\title{
Histological discrimination of idiopathic inflammatory bowel disease from other types of colitis
}

\author{
N Le Berre, D Heresbach, M Kerbaol, S Caulet, J F Bretagne, J Chaperon, \\ M Gosselin, M P Ramée
}

\begin{abstract}
Aims-To assess the value of histology in diagnosing inflammatory bowel disease (IBD) in colorectal biopsy specimens.

Methods-Retrospective, double blind evaluation of colorectal biopsy specimens from 41 patients with colitis ( 28 with ischaemic colitis and 13 with acute self-limited colitis) and 84 patients with IBD (42 with Crohn's disease and 42 with ulcerative colitis).

Results-The features distinguishing IBD from other forms of colitis included distorted architecture, lymphocyte and plasma cell infiltrate, excess of polymorphonuclear leucocytes, polymorphonuclear cryptitis, crypt abscesses, and basal lymphoid aggregates. The features discriminating between Crohn's disease and ulcerative colitis included an irregular or villous surface, distorted architecture, decrease in mucus content, and polymorphonuclear cryptitis. Using multivariate analysis, $90 \%$ of patients with Crohn's disease and $71 \%$ of those with ulcerative colitis were correctly classified, the former being strongly defined by epithelioid granulomas, microgranulomas and isolated giant cells, and the latter best defined by an irregular or villous surface, decrease in mucus content and crypt atrophy.
\end{abstract}

Conclusions-Examination of colorectal biopsy specimens is a reliable method for diagnosing IBD. In the absence of epithelioid granulomas, microgranulomas and isolated giant cells a diagnosis of Crohn's disease is based on the absence of histological criteria favouring ulcerative colitis. The histological spectrum of indeterminate colitis remains to be clarified. ( $f$ Clin Pathol 1995;48:749-753)

Keywords: Crohn's disease, ulcerative colitis, colorectal biopsy specimens.

Hepatogastroenterology D Heresbach J F Bretagne M Gosselin

Department of Public Health

M Kerbaol

J Chaperon

INSERM

M Kerbaol

Correspondence to: Dr N Le Berre.

Accepted for publication 21 February 1995 about 10 to $20 \%$ of colectomy specimens remain unclassified and are designated "indeterminate colitis". ${ }^{1}$

An early and accurate diagnosis is necessary for correct therapeutic management. In par-
Idiopathic inflammatory bowel disease (IBD) is divided into two major forms: ulcerativ colitis and Crohn's disease, the diagnosis of which is based on clinical, radiological, endo- ticular, it is important to distinguish between IBD and acute self-limited colitis. Moreover, a differential diagnosis between ulcerative colitis and Crohn's disease is required because their medical and surgical management may differ. ${ }^{23}$ The histopathological diagnosis of IBD should therefore be based on discriminating histological features which are sufficiently reproducible and suitable in routinely processed biopsy specimens. We performed a retrospective, double blind evaluation of a large number of histopathological features in colonic biopsy specimens from patients with clinically well defined IBD or non-relapsing colitis to assess the reproducibility of the histopathological features; to determine which features have the highest discriminative power in distinguishing IBD from non-relapsing colitis; and to determine which histological features are the most useful for distinguishing between ulcerative colitis and Crohn's disease.

\section{Methods}

Colorectal biopsy specimens from 125 patients were evaluated. Of the 84 patients with IBD, 42 had Crohn's disease (20 men and 22 women; mean age 34 years, range $15-78$ years), while the other 42 had ulcerative colitis (24 men and 18 women; mean age 39 years, range 19-77 years). The diagnoses of Crohn's disease or ulcerative colitis were based on well established clinical, endoscopic and radiological criteria. ${ }^{4}$ Pathological features were not taken into account. The control group comprised 41 patients: 28 patients with ischaemic colitis and 13 with acute self-limited, infectious colitis. The following criteria were required for the diagnosis of ischaemic colitis: a history of arteriopathy; clinical presentation consistent with ischaemic colitis (abdominal pain, diarrhoea, rectorragy); segmental colitis on endoscopic examination; spontaneous clinical resolution. The criteria for inclusion in the group with acute self-limited infectious colitis were: a first episode of acute diarrhoea; rapid resolution; stool culture or serodiagnosis of a pathogenic micro-organism; the typical endoscopic appearance of pseudomembranous colitis after recent antibiotic treatment; the detection of Clostridium difficile or its toxin in stools.

We reviewed 246 colonic biopsy specimens. Biopsy specimens were fixed in formaldehyde, embedded in paraffin wax, step-sectioned at $4 \mu \mathrm{m}$, and stained with haematoxylin and eosin and periodic acid-Schiff. At least 10 serial sec- 
Table 1 Histopathological features used in the evaluation of colonic biopsy specimens (see appendix for greater detail)

\begin{tabular}{ll}
\hline $\begin{array}{l}\text { Feature } \\
\text { number }\end{array}$ & Definition \\
\hline 1 & Inflammatory exudate \\
2 & Pseudomembranes \\
3 & Surface epithelium (intact/erosion or ulceration) \\
4 & Surface configuration (normal/irregular or villous) \\
5 & Neutrophils in surface epithelium \\
6 & Crypt architecture (normal/distorted) \\
7 & Crypt atrophy \\
8 & Mucus content (normal or increased/decreased) \\
9 & Dysplasia \\
10 & Paneth cell metaplasia \\
11 & Oedema \\
12 & Congested capillaries \\
13 & Lymphocyte and plasma cell infiltrate \\
14 & Excess of histiocytes \\
15 & Muciphages \\
16 & Excess of polymorphonuclear leucocytes \\
17 & Excess of neutrophils \\
18 & Excess of eosinophils \\
19 & Excess of mast cells \\
20 & Polymorphonuclear cryptitis \\
21 & Neutrophilic cryptitis \\
22 & Eosinophilic cryptitis \\
23 & Histiocytic cryptitis \\
24 & Epithelioid or giant cell cryptitis \\
25 & Crypt abscesses \\
26 & Basal lymphoid aggregates \\
27 & Epithelioid granulomas \\
28 & Microgranulomas \\
29 & Isolated giant cells \\
30 & Muscularis mucosae (normal/thickened) \\
31 & Disproportionate submucosal inflammation \\
32 & Distribution of inflammation (continuous/ \\
33 & discontinuous) \\
& Increase in the degree of inflammation (from \\
proximal to distal/from distal to proximal) \\
\hline &
\end{tabular}

tions from each biopsy specimen were examined by two independent pathologists with a special interest in gastrointestinal histopathology (NLB and MPR), who were unaware of the patients' clinical details. Thirty three histological features (table 1) were evaluated. Some of these features are self explanatory while others require further explanation (see appendix). The degree of agreement between observers was characterised using the $\kappa$ statistic: $\kappa$ is an index of interobserver agreement which has been corrected for chance. $\kappa$ values greater than 0.75 represent excellent agreement beyond chance, values below 0.4 represent poor agreement and values between 0.4 and 0.75 represent fair to good agreement. ${ }^{5}$ Sufficiently

Table 2 Histopathological features with sufficient reproducibility

\begin{tabular}{rll}
\hline $\begin{aligned} \text { Feature } \\
\text { number }\end{aligned}$ & Definition & \\
\hline 23 & Histiocytic cryptitis & $\kappa$ \\
24 & Epithelioid or giant cells cryptitis & 1 \\
33 & Increase in the degree of inflammation & 1 \\
27 & Epithelioid granulomas & 1 \\
32 & Distribution of inflammation & $0 \cdot 95$ \\
29 & Isolated giant cells & $0 \cdot 86$ \\
20 & Polymorphonuclear cryptitis & $0 \cdot 85$ \\
4 & Surface configuation & $0 \cdot 77$ \\
28 & Microgranulomas & $0 \cdot 75$ \\
13 & Lymphocyte and plasma cell infiltrate & $0 \cdot 75$ \\
7 & Crypt atrophy & $0 \cdot 68$ \\
2 & Pseudomembranes & $0 \cdot 67$ \\
3 & Surface epithelium & $0 \cdot 66$ \\
21 & Neutrophilic cryptitis & $0 \cdot 66$ \\
25 & Crypt abscesses & $0 \cdot 66$ \\
16 & Excess of polymorphonuclear leucocytes & $0 \cdot 64$ \\
6 & Crypt architecture & $0 \cdot 64$ \\
31 & Disproportionate submucosal inflammation & $0 \cdot 63$ \\
8 & Mucus content & $0 \cdot 63$ \\
14 & Excess of histiocytes & $0 \cdot 62$ \\
11 & Oedema & $0 \cdot 60$ \\
30 & Muscularis mucosae & $0 \cdot 57$ \\
26 & Basal lymphoid aggregates & $0 \cdot 53$ \\
9 & Dysplasia & $0 \cdot 52$ \\
17 & Excess of neutrophils & $0 \cdot 49$ \\
12 & Congested capillaries & $0 \cdot 47$ \\
19 & Excess of mast cells & $0 \cdot 45$ \\
1 & Inflammatory exudate & $0 \cdot 45$ \\
\hline & & $0 \cdot 44$ \\
\hline
\end{tabular}

reproducible histological features were analysed by univariate analysis using the $\chi^{2}$ test; $\mathrm{p}<0.05$ was considered significant. Multivariate analysis comprised factorial correspondence analysis with hierarchical ascending classification. ${ }^{6}$ This method is based on the measurement of the similarity between individuals in mathematical terms and then automatically groups these individuals. Thus, it reduces the subjective element of the classification process. No prior assumption is made that a population is divisible into groups. Where it is possible to divide patients into groups according to their attributes, discriminant analysis can be applied to calculate which features allocate a patient most accurately to each group, and therefore to ascertain which features are the most important in effecting this separation. ${ }^{7}$

\section{Results}

According to the $\kappa$ value, 28 of 33 histological features were sufficiently reproducible (table 2).

The 11 histological features with a significant discriminant value in distinguishing IBD from non-relapsing colitis are presented in table 3 . The most discriminative features $(p<0.0001)$ favoured IBD with a good predictive probability (70 to $90 \%$ ).

Fourteen histological features discriminated between Crohn's disease and ulcerative colitis (table 4). The most discriminative $(\mathrm{p}<0.0001)$ favoured ulcerative colitis over Crohn's disease with a predictive probability of 61 to $97 \%$. Three features favoured Crohn's disease over ulcerative colitis: epithelioid granulomas $(p<0.01)$, microgranulomas and an excess of histiocytes $(p<0 \cdot 05)$.

Factorial correspondence analysis of the 125 patients yielded two large groups of patients. Comparison of these groups with the clinical diagnosis showed that one mainly contained patients with Crohn's disease, whereas the other contained patients with either ulcerative colitis or non-relapsing colitis (ischaemic colitis or acute self-limited colitis) with no demarcation between these diagnoses (data not shown). The number of histological features recorded was too large to allow features characteristic of the groups to be determined. The analysis was therefore limited to those patients with IBD. The automatic hierarchical ascending classification yielded two groups of patients: one group contained $90 \%$ of the patients with Crohn's disease, while the second contained $71 \%$ of the patients with ulcerative colitis. The patients could be separated into these two groups using nine criteria (table 5).

A graphical representation of factorial analysis of patients with IBD according to these nine histological features is shown in the figure. According to the horizontal axis, one group of patients, most of them with Crohn's disease, is located on the right of the graph. This group is delineated by epithelioid granulomas and microgranulomas. Patients with ulcerative colitis are grouped on the left of the graph and are delineated by a decrease in mucus content, 
Table 3 Histological features predictive of IBD

\begin{tabular}{rlllll}
\hline $\begin{array}{l}\text { Feature } \\
\text { number }\end{array}$ & Definition & $\begin{array}{l}\text { IBD } \\
(\%)\end{array}$ & $\begin{array}{l}\text { Non-relapsing } \\
\text { colitis (\%) }\end{array}$ & p value & $\begin{array}{l}\text { Predictive } \\
\text { probability (\%) }\end{array}$ \\
\hline 6 & Crypt achitecture (distorted) & 77 & 32 & $<0 \cdot 0001$ & 71 \\
13 & Lymphoplasma cell infiltrate & 82 & 17 & $<0 \cdot 0001$ & 83 \\
16 & Excess of polymorphonuclear leucocytes & 92 & 39 & $<0 \cdot 0001$ & 70 \\
20 & Polymorphonuclear cryptitis & 65 & 7 & $<0 \cdot 0001$ & 90 \\
25 & Crypt abscesses & 51 & 7 & $<0 \cdot 0001$ & 88 \\
26 & Basal lymphoid aggreggates & 62 & 17 & $<0 \cdot 0001$ & 78 \\
3 & Surface (erosion or ulceration) & 93 & 76 & $<0.01$ & 55 \\
4 & Surface (irregular or villous) & 55 & 22 & $<0.01$ & 71 \\
14 & Excess of histiocytes & 46 & 15 & $<0.05$ & 69 \\
19 & Excess of mast cells & 33 & 15 & $<0.05$ & 88 \\
28 & Microgranulomas & 15 & 2 & & \\
\hline
\end{tabular}

Table 4 Histological features discriminating between Crohn's disease and ulcerative colitis

\begin{tabular}{|c|c|c|c|c|c|}
\hline $\begin{array}{l}\text { Feature } \\
\text { number }\end{array}$ & Definition & $\begin{array}{l}\text { Crohn's } \\
\text { diesease (\%) }\end{array}$ & $\begin{array}{l}\text { Ulcerative } \\
\text { colitis (\%) }\end{array}$ & $p$ value & $\begin{array}{l}\text { Predictive } \\
\text { probability (\%) }\end{array}$ \\
\hline 4 & Surface (irregular or villous) & 21 & 88 & $<0.0001$ & $81^{*}$ \\
\hline 6 & Crypt architecture (distorted) & 60 & 95 & $<0.0001$ & $61^{*}$ \\
\hline 8 & Mucus content (decreased) & 2 & 62 & $<0.0001$ & $97^{*}$ \\
\hline 20 & Polymorphonuclear cryptitis & $4 \overline{5}$ & 86 & $<0.0001$ & $66^{*}$ \\
\hline 7 & Crypt atrophy & 10 & 43 & $<0.01$ & $68^{*}$ \\
\hline 16 & Excess of polymorphonuclear leucocytes & 86 & 98 & $<0.01$ & $53^{*}$ \\
\hline 25 & Crypt abscesses & 33 & 69 & $<0.01$ & $68^{*}$ \\
\hline 27 & Epithelioid granulomas & 21 & 0 & $<0.01$ & $100^{* *}$ \\
\hline 1 & Inflammatory exudate & 38 & 62 & $<0.05$ & $62^{*}$ \\
\hline 3 & Surface (erosion or ulceration) & 86 & 100 & $<0.05$ & $53^{*}$ \\
\hline 12 & Congested capillaries & 7 & 24 & $<0.05$ & $77^{*}$ \\
\hline 13 & Lymphoplasma cell infiltrate & 71 & 93 & $<0.05$ & $57^{*}$ \\
\hline 14 & Excess of histiocytes & 57 & 36 & $<0.05$ & $61^{* *}$ \\
\hline 28 & Microgranulomas & 24 & 7 & $<0.05$ & $77^{* *}$ \\
\hline
\end{tabular}

*Ulcerative colitis; ${ }^{* *}$ Crohn's disease.

crypt atrophy and an irregular or villous surface. Interpretation of the vertical axis is more difficult; while patients with ulcerative colitis are clustered in the central area of the vertical axis, those with Crohn's disease are scattered.

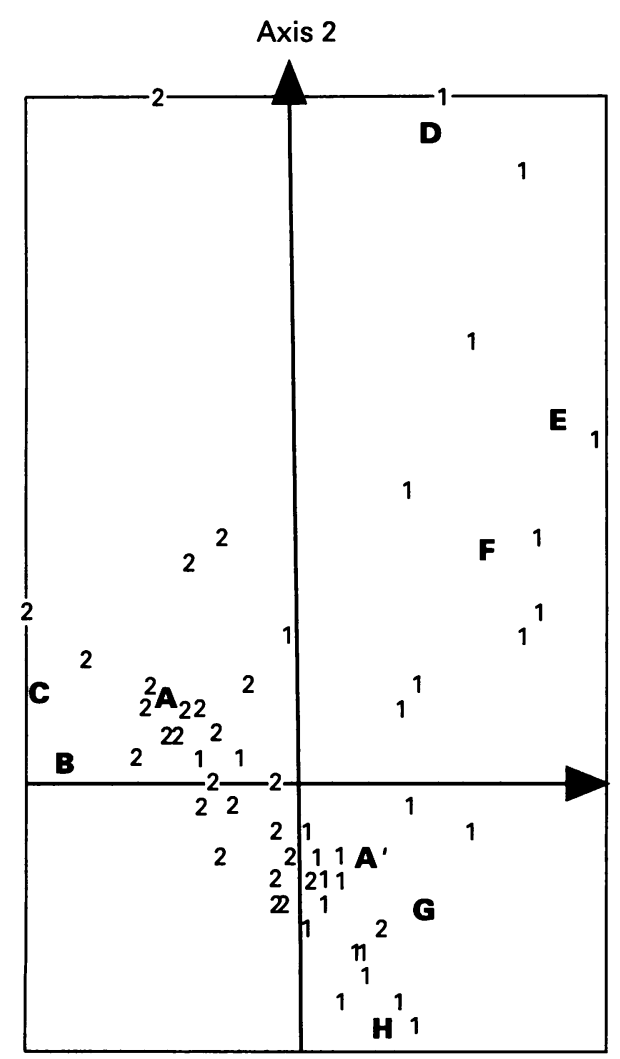

Graphical representation of factorial correspondence analysis of patients with Crohn's disease (1) or ulcerative colitis (2). $A$, irregular/villous surface; $A$, normal surface; $B$, atrophy; $C$, decrease in mucus content; $D$, isolated giant cells; $E$, epithelioid granulomas; $F$, microgranulomas; $G$, polymorphonuclear cryptitis absent; $H$, polymorphonuclear infiltrate absent.
Only $30 \%$ of the patients with Crohn's disease are located in the positive area of the vertical axis, delineated by isolated giant cells, epithelioid granulomas and microgranulomas. Conversely, most of the patients with Crohn's disease are located in the negative area of the vertical axis, delineated by absence of polymorphonuclear infiltrate, absence of polymorphonuclear cryptitis and normal surface configuration.

\section{Discussion}

This retrospective study has shown that, according to the $\kappa$ statistic, 28 of 33 histological features were sufficiently reproducible. These results differ from those of previous similar studies: Seldenrijk et $a l^{8}$ and Giard et $a l^{9}{ }^{9}$ respectively, found that 16 and five features were sufficiently reproducible, but these authors studied the degree of interobserver agreement between, respectively, four and three pathologists. More recently, Theodossi et $a^{10}$ studied the range of agreement between 10 observers: they showed that there was considerable disagreement in reaching a diagnosis, but they also found 15 of 41 evaluated features with agreement measures significantly better

Table 5 Histological criteria determining the separation of the groups of patients on multivariate analysis

\begin{aligned} & $\begin{array}{l}\text { Feature } \\ \text { number }\end{array}$ Definition \\ & \hline 4 Surface configuration \\ & 7 Crypt atrophy \\ & 8 Mucus content \\ & 25 Crypt abscesses \\ & 27 Epithelioid granulomas \\ & 28 Microgranulomas \\ & 29 Isolated giant cells \\ & 16 Excess of polymorphonuclear leucocytes \\ & 20 Polymorphonuclear cryptitis \\ & \hline\end{aligned}


than expected by chance after classifying these features on a present/absent scale. The very good reproducibility we found in our study, however, is probably overestimated because the biopsy specimens were evaluated by only two observers.

The univariate analysis yielded 11 histological features which could be used to distinguish IBD from non-relapsing colitis. We agree with previous studies ${ }^{112}$ that the diagnosis of non-relapsing colitis is best based on the absence of the histological features of IBD. Some of our findings have been reported by other authors: similar studies have shown that crypt architectural abnormalities, ${ }^{810-15}$ an increase in lymphocyte and plasma cell infiltrate 8101315 and basal lymphoid aggregates $^{1115}$ are highly predictive of IBD. Our finding that crypt abscesses are also diagnostic of IBD is more controversial: Surawicz et $a l^{11}$ found that crypt abscesses were predictive of acute self-limited colitis rather than of IBD, but more recently another study showed that they were more common in the latter than in the former. ${ }^{15}$ Surprisingly, we found that an excess of polymorphonuclear cells in the lamina propria and polymorphonuclear cryptitis were both highly predictive of IBD; however, our non-relapsing colitis group mainly included patients with ischaemic colitis which is perhaps the reason for this finding. We agree with the findings of Allison et $a^{13}$ that surface erosions are also diagnostic of IBD. Moreover, we confirm the conclusions of similar studies that a villous or irregular mucosal surface is highly predictive of IBD. ${ }^{11215} \mathrm{We}$ also found that an excess of mast cells and histiocytes in the lamina propria is significantly more common in IBD. Surprisingly, we found that microgranulomas are diagnostic of IBD, whereas epithelioid granulomas are not. Epithelioid granulomas were found in two patients with acute self-limited colitis $(4.9 \%$ of patients with non-relapsing colitis) and in nine $(10.7 \%)$ patients with Crohn's disease. This difference was not significant.

Fourteen features showed a significant discriminant power in distinguishing Crohn's disease from ulcerative colitis. Our finding that a decrease in mucus content, a villous or irregular aspect to the mucosal surface, distorted crypt architecture, and polymorphonuclear cryptitis highly are predictive of ulcerative colitis rather than Crohn's disease confirm the results of other studies. ${ }^{10116}$ Epithelioid granulomas and microgranulomas, predictive of Crohn's disease, were found in, respectively, $21 \%$ and $24 \%$ of the biopsy specimens taken from patients with Crohn's disease. Moreover, we confirm the findings of Seldenrijk et $a l^{8}$ that an excess of histiocytes is predictive of Crohn's disease.

As yet, factorial correspondence analysis has not been widely applied to histopathological diagnosis. In the present study, the analysis limited to those patients with IBD yielded two main groups, containing, respectively, $90 \%$ and $71 \%$ of patients with Crohn's disease and ulcerative colitis. Using multivariate analysis, we found that an irregular or villous surface, a decrease in mucus content and crypt atrophy is the best combination for discriminating between ulcerative colitis and Crohn's disease. Unsurprisingly, epithelioid granulomas and isolated giant cells strongly suggest a diagnosis of Crohn's disease. We found that microgranulomas are also diagnostic of Crohn's disease rather than of ulcerative colitis. Interestingly, patients with Crohn's disease were divided into two smaller clusters, one of which was delineated by the classic features of Crohn's disease-that is, epithelioid granulomas, giant cells and microgranulomas. The second cluster was delineated by negative features such as the absence of polymorphonuclear infiltrate and polymorphonuclear cryptitis, and a normal mucosal surface. The interpretation of the data for this second group of patients with Crohn's disease is more difficult because our retrospective study did not allow us to control for disease duration, the severity of clinical activity or whether the patients were undergoing treatment when the biopsy specimens were taken. Our findings agree with those of Theodossi et $a l^{10}$ that the diagnosis of Crohn's disease is often difficult in the absence of highly predictive features; however, the presence of chronic inflammation in the absence of features predictive of ulcerative colitis suggests a diagnosis of Crohn's disease.

In conclusion, our study validates the use of colorectal biopsy specimens for the diagnosis of IBD. Discriminant histological features distinguishing IBD from non-relapsing colitis and Crohn's disease from ulcerative colitis were identified on univariate analysis. Moreover, using multivariate analysis, we were able to classify correctly $90 \%$ and $71 \%$, respectively, of patients with Crohn's disease and ulcerative colitis, using only nine histological features. The problem of so-called "indeterminate colitis" remains unresolved. It would be interesting to apply these histological criteria to patients with "indeterminate colitis" and assess their reliability on clinical follow up.

1 Price AB. Overlap in the spectrum of non-specific inflammatory bowel disease- "colitis indeterminate". $f$ Clin Pathol 1978;31:567-77.

2 Hyman NH, Fazio VW, Tuckson WB, Lavery IC. Consequences of ileal pouch-anal anastomosis for Crohn's sequences of ileal pouch-anal anastom.

3 Koltun WA, Schoetz DJ Jr, Roberts PL, Murray JJ, Coller JA, Veidenheimer MC. Indeterminate colitis predispose to perineal complications after ileal pouch-anal anastomosis. Dis Colon Rectum 1991;34:857-60.

4 Lennard-Jones JE. Classification of inflammatory bowe disease. Scand $\mathcal{f}$ Gastroenterol 1989;24(Suppl 170):2-6.

5 Landis JR, Koch GG. The measurement of observer agreement for categorical data. Biometrics 1977;33:159-74.

6 Fenelon JP, ed. Qu'est-ce que l'analyse des données? Paris: Leforen, 1981.

7 Hywel Jones J, Lennard-Jones JE, Morson BC, Chapman M, Sackin MJ, Sneath PHA, et al. Numerical taxonomy and discriminant analysis applied to non-specific colitis. and discriminant analysis app

8 Seldenrijk CA, Morson BC, Meuwissen SGM, Schipper NW, Lindeman J, Meijer CJLM. Histopathological evaluation of colonic mucosal biopsy specimens in chronic inflammatory bowel disease: diagnostic implications. Gut 1991;32:1514-20.

9 Giard RWM, Hermans J, Ruiter DJ, Hoedemaeker PJ. Variations in histopathogical evaluation of non-neoplastic colonic mucosal abnormalities; assessment and clinica significance. Histopathology 1985;9:535-41.

10 Theodossi A, Spiegelhalter DJ, Jass J, Firth J, Dixon M, Leader $\mathrm{M}$, et al. Observer variation and discriminatory value of biopsy features in inflammatory bowel disease. Gut 1994;35:961-8.

11 Surawicz CM, Belic L. Rectal biopsy helps to distinguish acute self-limited colitis from idiopathic inflammatory bowel disease. Gastroenterology 1984;86:104-13.

12 Schumacher G, Kollberg B, Sandstedt B. A prospective 
study of first attacks of inflammatory bowel disease and infectious colitis. Histologic course during the 1st year after presentation. Scand $\mathscr{\mathcal { F }}$ Gastroenterol 1994;29:318-32.

13 Allison MC, Hamilton-Dutoit SJ, Dhillon AP, Pounder RE The value of rectal biopsy in distinguishing self-limited colitis from early inflammatory bowel disease. $Q \mathcal{F}$ Med colitis from early

14 Schumacher G, Standstedt B, Mollby R, Kollberg B. Clinical and histologic features differentiating non-relapsing colitis from first attacks of inflammatory bowel disease. Scand $\mathcal{F}$ Gastroenterol 1991;26:151-61.

15 Surawicz CM, Haggit RC, Husseman M, McFarland LV. Mucosal biopsy diagnosis of colitis: acute self-limited colitis and idiopathic inflammatory bowel disease. Gastroenterology 1994;107:755-63.

16 McCormick DA, Horton LWL, Mee AS. Mucin depletion in inflammatory bowel disease. $\mathcal{F}$ Clin Pathol 1990;43: 143-6.

17 Riddel RH, Goldman H, Ransohoff DF, Appelman HD, Fenaglio CM, Haggitt RC, et al. Dysplasia in inflammatory bowel disease: standardized classification with provisional clinical applications. Hum Pathol 1983;14:931-68.

\section{Appendix}

DEFINITIONS OF HISTOLOGICAL FEATURES

Feature 3-An erosion or an ulceration is diagnosed if neutrophils are present in the base of the area of loss of the surface epithelium.

Feature 4-A villous mucosal surface is a surface contour of broad villus-like projections with a villus : crypt ratio of more than $1 \cdot 5$. A mucosal surface with a villus : crypt ratio between 1 and 1.5 is regarded as an irregular surface. ${ }^{8}$

Feature 6-Crypt architecture is normal if the crypts are parallel and the space between them is not excessive (see definition of "atrophy"). It is distorted if the crypts are dilatated, tortuous, or branching in a well orientated biopsy specimen. $^{811}$

Feature 7-Crypt atrophy is diagnosed if the crypts are shortened and more widely spaced, and if there is a gap between the bottom of the crypts and the muscularis mucosae.
Feature 9-Dysplasia is defined as an unequivocal neoplastic alteration of the epithelium, according to Riddell's classification. ${ }^{17}$ Feature 10-Paneth cells are normally restricted to the ascending colon.

Feature 13-An increase in the number of lymphocytes and plasma cells is diagnosed only if there is an obvious increase in their number.

Features 17 to $19-$ Neutrophil and eosinophil excess is diagnosed if there is an unequivocal increase in the number of such cells in the lamina propria, according to the pathologist.

Features 20 to 24 -Cryptitis is defined as a migration of inflammatory cells into the crypt epithelium with focal lysis of epithelial cells.

Feature 25-A crypt abscess is a chain of neutrophils extending from the lamina propria through the crypt epithelium into the lumen of the crypt.

Feature 26-Basal lymphoid aggregates are nodular collections of lymphocytes without reactive centres, located between the muscularis mucosae and the crypts.

Feature 27-An epithelioid granuloma is a collection of at least five epithelioid cells with or without accompanying giant cells, and without caseating necrosis or foreign bodies.

Feature 28-A microgranuloma is an aggregate of histiocytes and lymphocytes; giant cells are absent.

Feature 29-Giant cells contain multiple nuclei and homogeneous, fine, powdery cytoplasm without foreign bodies.

Feature 31-Inflammation is disproportionate if the submucosa contains a denser infiltrate than the mucosa. 